\title{
Stress Hyperglycaemia Indicates Embolus Size and Localization in Patients with Acute Pulmonary Embolism
}

\author{
Velimir Altabas $\mathbb{D}^{1},{ }^{1,2}$ Lucija Pukec, ${ }^{1}$ Stella Mlinarić, ${ }^{1}$ Hrvoje Pintarić, ${ }^{3}$ and Aljoša Šikić ${ }^{3}$ \\ ${ }^{1}$ School of Medicine, University of Zagreb, Zagreb, Croatia \\ ${ }^{2}$ Clinic for Internal Medicine, Department of Endocrinology, Diabetes and Metabolic Diseases, \\ Sestre Milosrdnice University Hospital Center, Zagreb, Croatia \\ ${ }^{3}$ Emergency Department, Sestre Milosrdnice University Hospital Center, Zagreb, Croatia \\ Correspondence should be addressed to Velimir Altabas; velimir.altabas@gmail.com
}

Received 4 May 2020; Accepted 17 June 2020; Published 8 July 2020

Academic Editor: Marco Faustini-Fustini

Copyright (c) 2020 Velimir Altabas et al. This is an open access article distributed under the Creative Commons Attribution License, which permits unrestricted use, distribution, and reproduction in any medium, provided the original work is properly cited.

\begin{abstract}
Objective. Acute pulmonary embolism is a life-threatening form of venous thromboembolism often causing stress hyperglycaemia. The aim of this study was to determine the prognostic value of stress hyperglycaemia in acute pulmonary embolism, providing new insights into the presumed embolus size and localization, clinical parameters (Pulmonary Embolism Severity Index, PESI), and in-hospital mortality. Design and Methods. Among a total of 95,454 patients referred to the Emergency Department of the Sestre Milosrdnice University Hospital Centre between 2014 and 2016, all patients with acute pulmonary embolism were included into this observational cohort study. The study group consisted of 190 patients aged 25-96. Relevant patient history, clinical data, and laboratory findings were collected during the entire hospitalization period. Data were analyzed for the entire group of patients, as well as separately for patients without diabetes, using the Fisher exact test and logistic regression. Results. Analysis of embolus localization as an indirect parameter of embolus size showed that patients with stress hyperglycaemia more often had emboli located in proximal parts of the pulmonary circulation (i.e., main artery or lobar branches) $(p<0.05)$. Furthermore, stress hyperglycaemia correlated with PESI score and diabetes $(p<0.05)$ in the entire patient group. Stress hyperglycaemia showed independent association with in-hospital mortality in patients $(p<0.05)$. Conclusion. Stress hyperglycaemia in patients with acute pulmonary embolism is associated with embolus localization in larger arteries of the pulmonary circulation and higher PESI score and therefore could serve as an independent in-hospital mortality predictor.
\end{abstract}

\section{Introduction}

Acute pulmonary embolism (APE) is a life-threatening form of venous thromboembolism (VTE) often associated with stress hyperglycaemia (SH). Risk factors for APE are divided into two groups, environmental and genetic. Patient' history of deep vein thrombosis (DVT), malignancy, patient age, neurological disease, trauma or fracture, immobilization, and major surgery are closely related to the occurrence of VTE, including pulmonary embolism (PE) [1-3].

Clinical presentation of APE is diverse and does not always correlate with radiographic findings; however, multislice computed tomography angiography (MSCTA) is the gold standard in diagnosing $\mathrm{PE}$ with a sensitivity of
$83-100 \%$ and specificity of $89-96 \%$. Other procedures, such as Pulmonary Embolism Severity Index (PESI) scoring, are used to estimate clinical outcomes [4]. PESI is a prognostic model for APE that includes 11 clinical criteria that predict a 30-day patient mortality [5].

$\mathrm{SH}$ refers to transient elevated plasma glucose (PG), most commonly detected during hospital admission. It is a frequent laboratory finding in severe acute medical and surgical conditions in patients without diabetes mellitus (DM), although SH may also occur in patients with formerly well-controlled DM [6-8]. There is a prevalent misconception that $\mathrm{SH}$ equals diabetes, but it has to be stressed that these are two separate yet, similar conditions. Stress hyperglycemia occurs secondary to an acute disease and will 
disappear over time in the majority of patients, while diabetes is a longstanding condition resulting in chronic complications.

The onset mechanisms of hyperglycaemia in DM and $\mathrm{SH}$ only partially overlap. The pathophysiology of $\mathrm{SH}$ is based on the neuroendocrine and inflammatory response to acute stressful events. Activation of the hypothalamus-pituitaryadrenal axis and the sympathetic nervous system results in increased secretion of counterregulatory hormones (i.e., cortisol, glucagon, adrenaline, noradrenaline, and growth hormone) that leads to changes in carbohydrate metabolism [8]. As part of the stress response, mediators of inflammation are secreted, reducing glucose utilization in peripheral tissues and stimulating the release of counterregulatory hormones, which enhances hyperglycaemia, cytokine secretion, inflammatory response, and oxidative stress and closes the vicious cycle [6]. However, hyperglycaemia in diabetes is mostly caused by defects in insulin secretion, insulin resistance, and functional disruptions of various organs (e.g., the brain, gut, and kidney) [9].

Previous research has demonstrated the occurrence of $\mathrm{SH}$ in myocardial infarction, stroke, trauma, burns, sepsis, postoperative, and in APE [7, 8, 10-12], but due to nonharmonized criteria for $\mathrm{SH}$, this condition is still not well understood. For example, there is no universally accepted threshold for the blood glucose level to define SH, which complicates calculating the prevalence of $\mathrm{SH}$ and its consequences $[13,14]$. According to a systematic literature review by Olariu et al., SH has been consistently associated with poor disease outcomes [13]. In APE, elevated admission PG has been shown to be an independent risk factor for a 30day mortality [7]. A few studies have shown that for patients with acute myocardial infarction and the patients with $\mathrm{SH}$ without previous diagnosis of DM have more severe outcomes than those with DM [15].

The aim of this study was to investigate correlations between SH, embolus size and localization, clinical parameters, and in-hospital mortality in APE.

To our best knowledge, there is no evidence in current literature about $\mathrm{SH}$ indicating specific radiological findings that may impact prognostic scores and in-hospital mortality in patients with APE.

\section{Materials and Methods}

This observational cohort study was conducted in concordance with the Declaration of Helsinki as revised in 2008, and it was approved by the ethical boards of the Sestre Milosrdnice University Hospital Centre and of the School of Medicine at the University in Zagreb.

Between January 01, 2014 and December 31, 2016, there were 95,454 patients admitted to the Emergency Department at Sestre Milosrdnice University Hospital Centre in Zagreb. All patients underwent standard admission protocols (i.e., medical history, physical examination, and basic laboratory tests, including PG and electrocardiogram). All patients with suspected APE underwent MSCTA to confirm the diagnosis. Among them, 253 patients, aged
25-96, were hospitalized for acute PE in Sestre Milosrdnice University Hospital Centre in Zagreb and were recruited into the study. Relevant patient information was collected from electronic records.

Patients whose symptoms lasted for 7or more days $(n=48)$, those who did not get MSCTA confirmation because of sudden death $(n=3)$, and those with radiographic signs of chronic PE $(n=2)$ were excluded from the study. Based on radiological findings, localization of the embolus was used to indirectly define the approximate embolus size, assuming that large emboli cannot reach vessels with a smaller diameter without dissolution. Emboli found in the main or in the lobar branches of pulmonary arteries were considered as large, while small emboli were those found in segmental or subsegmental branches. Final PESI score was assigned by summing points for each characteristic as in the original PESI study [16]. To calculate the PESI score, data on age, sex, heart failure, chronic lung disease, malignancy, mental status, systolic pressure, heart rate, respiratory rate, body temperature, and oxygen saturation at admission were collected. According to age, patients were divided into younger patient group ( $<65$ years old) and older patient group (65 years and older), as in the original PESI study. In statistics, PESI classes 1 and 2 were considered low class, while classes 3, 4, and 5 were considered high class. Patients who did not have all anamnestic or clinical data necessary for PESI scoring were excluded $(n=10)$.

The study sample consisted of 190 patients, 154 nondiabetics and 36 diabetics.

PG values used in this study were the earliest ones measured in the emergency department. The cut-off value used for $\mathrm{SH}$ in this study was $\geq 7.8 \mathrm{mmol} / \mathrm{L}$, as used by the American Diabetes Association for elevated blood glucose in chronic conditions such as prediabetes and diabetes.

Because of chronic hyperglycaemia in patients with DM with fair blood glucose control, criteria for $\mathrm{SH}$ should be different for this subgroup of diabetic patients, and therefore, data on diabetic patients should be interpreted with more caution.

Because of the small sample size, metabolic changes, possible previous fair blood glucose control, and therapy interference, data on patients with DM were not specifically analyzed.

Finally, data on in-hospital mortality were collected.

Statistical analysis was done using descriptive statistics, the Fisher exact test (FET) for the univariate analysis, and logistic regression for the multivariate analysis. Significance level used in tests was 0.05. Analysis was done using MS Office 2013 and MedCalc ${ }^{\circledR}$ 19.1.7 (MedCalc, Ostend, Belgium).

\section{Results}

Patient characteristics are shown in Table 1.

In this study, the statistical analysis was done for the entire patient group $(n=190)$, along with a subgroup of patients without DM $(n=154)$. The data were compared to point out the differences depending on inclusion or exclusion of diabetic patients. 
Table 1: Patient characteristics.

\begin{tabular}{|c|c|c|c|}
\hline & All patients (190) & Nondiabetics (154) & Diabetics (36) \\
\hline \multicolumn{4}{|l|}{ Demographics } \\
\hline Age (median, range) & $76(82-64)$ & $75.5(82-62)$ & $79(83-68.5)$ \\
\hline Age $(<65$ years $)$ & $49(25.79 \%)$ & $43(27.92 \%)$ & $6(16.67 \%)$ \\
\hline Men, $n(\%)$ & $70(36.84 \%)$ & $57(37.01)$ & $13(36.11)$ \\
\hline \multicolumn{4}{|l|}{ Medical history $(n, \%)$} \\
\hline Hypertension & $109(57.37)$ & $79(51.30)$ & $30(83.33)$ \\
\hline Diabetes mellitus & $36(18.95)$ & - & - \\
\hline Smoking & $35(18.42)$ & $31(20.13)$ & $4(11.11)$ \\
\hline History of thromboembolism & $29(15.26)$ & $24(15.58)$ & $5(13.89)$ \\
\hline Immobility & $32(16.84)$ & $23(14.94)$ & $9(25.00)$ \\
\hline History of malignancy & $51(26.84)$ & $41(26.62)$ & $10(27.78)$ \\
\hline History of heart failure & $8(4.21)$ & $6(3.90)$ & $2(5.56)$ \\
\hline History of COPD & $25(13.15)$ & $22(14.29)$ & $3(8.33)$ \\
\hline \multicolumn{4}{|l|}{ Clinical admission data $(n, \%)$} \\
\hline BMI $\left(\mathrm{kg} / \mathrm{m}^{2}\right)$ & $28.39( \pm 5.88)$ & $28.03( \pm 5.77)$ & $27.88( \pm 9.98)$ \\
\hline Stress hyperglycaemia & $89(46.84)$ & $62(40.26)$ & $27(75.00)$ \\
\hline Respiration $\geq 30 / \mathrm{min}$ & $22(11.58)$ & $17(11.04)$ & $5(13.89)$ \\
\hline Saturation $\mathrm{O}_{2}<90 \%$ & $82(43.16)$ & $63(40.91)$ & $19(52.78)$ \\
\hline Axillary temperature $<36^{\circ} \mathrm{C}$ & $1(0.01)$ & $1(0.64)$ & 0 \\
\hline Altered mental status & $20(10.53)$ & $17(11.04)$ & $3(8.33)$ \\
\hline \multicolumn{4}{|l|}{ Embolus localization } \\
\hline Main and lobar branches & $137(72.11)$ & $111(72.08)$ & $26(72.22)$ \\
\hline Segmental and subsegmental branches & $53(27.89)$ & $43(27.92)$ & $10(27.78)$ \\
\hline \multicolumn{4}{|l|}{ PESI class $(n, \%)$} \\
\hline Low & $55(28.95)$ & $49(31.82)$ & $6(16.67)$ \\
\hline High & $135(71.05)$ & $105(68.18)$ & $30(83.33)$ \\
\hline
\end{tabular}

According to the Fisher exact test for both the nondiabetics and the entire patient group, $\mathrm{SH}$ was shown to be associated with the PESI class (Table 2). This association remained significant after logistic regression in the group of patients without diabetes $(p=0.0075)$. In the entire patient group, $\mathrm{SH}$ was additionally independently associated with $\operatorname{DM}(p=0.0004)$ and embolus localization $(p=0.0248)$. After logistic regression, diabetes $(p=0.0009)$, embolus location $(p=0.0161)$, and PESI class $(p=0.0048)$ showed independent association with $\mathrm{SH}$.

A total of 25 patients died during hospitalization, 20 diabetics and 5 nondiabetics.

$\mathrm{SH}$ and patient history of malignancy were associated with in-hospital mortality by using the Fisher exact test in patients without diabetes. In the entire patient group, age group, $\mathrm{SH}$, and PESI class were associated with mortality after FET.

After logistic regression, independent association with in-hospital mortality in patients without DM was shown for SH $(p=0.0084)$ and patient history of malignancy $(p=0.0230)$. In the entire patient group, $\mathrm{SH}$ showed significant association with in-hospital mortality $(p=0.0495)$ after logistic regression (Table 3).

\section{Discussion}

In general, the main problem in $\mathrm{SH}$ studies is defining cutoff values. Among different studies, these values range from $6.1 \mathrm{mmol} / \mathrm{L}$ to $11.1 \mathrm{mmol} / \mathrm{L}$, which decreases reliability and complicates comparison of results. It was recommended that guidelines about $\mathrm{SH}$ values for this clinical entity should be made, but they still do not exist. Furthermore, in contrast to a vast quantity of research regarding cardiovascular outcomes related to stress hyperglycemia, there is only little evidence about $\mathrm{SH}$ and acute $\mathrm{PE}[7,17-19]$.

The prevalence of $\mathrm{SH}$ in acute $\mathrm{PE}$ in this study (46.84\%) is higher than the prevalence reported by Scherz et al. (33.58\%) for the $7.8 \mathrm{mmol} / \mathrm{L}$ cut-off value [7]. The higher value is probably due to the more advanced age and a higher prevalence of diabetes among patients in this study.

In the entire group of patients, logistic regression showed that DM and PESI score are risk factors for developing $\mathrm{SH}$, confirming results from previous studies [7, 20].

$\mathrm{SH}$ correlated well with PESI scores, which is a prognostic indicator combining several clinical parameters.

Furthermore, in the entire study population, the embolus localization as an indirect measure of embolus size was also shown to be an independent risk factor for developing $\mathrm{SH}$. These findings fit perfectly into the thesis that more profound stress is due to more massive embolization and more elevated admission glycaemia compared to embolization of more distal smaller vessels. However, further investigation will be needed to prove this concept in patients without diabetes.

The PESI model, commonly used to predict the 30-day mortality, showed no significance after logistic regression with in-hospital mortality for any patient group. In contrast to insignificant PESI statistic outcomes, $\mathrm{SH}$ showed independent association with in-hospital mortality in both patient groups, indicating that it could be 
TABle 2: Association of stress hyperglycaemia with patient characteristics.

\begin{tabular}{|c|c|c|c|c|}
\hline & \multicolumn{2}{|c|}{ FET (univariate analysis) } & \multicolumn{2}{|c|}{ Logistic regression (multivariate analysis) } \\
\hline & $p$ & Odds ratio $(95 \% \mathrm{CI})$ & $p$ & Odds ratio $(95 \% \mathrm{CI})$ \\
\hline \multicolumn{5}{|l|}{ Patients without DM (154) } \\
\hline Sex & 0.2336 & $1.5926(0.806-3.1467)$ & - & - \\
\hline Age & 0.0669 & $2.1175(0.9862-4.5464)$ & - & - \\
\hline Diabetes mellitus & - & & - & - \\
\hline Thromboembolic history & 1.0000 & $1.0714(0.4426-2.5938)$ & - & - \\
\hline Malignancy & 0.3601 & $1.4059(0.6831-2.8936)$ & - & - \\
\hline Embolus localization & 0.1434 & $1.8238(0.8607-3.8647)$ & - & - \\
\hline PESI class & 0.0022 & $2.8700(1.4639-5.6265)$ & 0.0075 & $2.8030(1.3169-5.9662)$ \\
\hline \multicolumn{5}{|l|}{ All patients (190) } \\
\hline Sex & 0.2925 & $1.4138(0.7797-2.5637)$ & - & - \\
\hline Age & 0.0670 & $1.9642(1.0005-3.8561)$ & - & - \\
\hline Diabetes mellitus & 0.0004 & $4.4516(1.9601-10.1101)$ & 0.0009 & $4.2590(1.8136-10.0015)$ \\
\hline Thromboembolic history & 0.6867 & $1.5297(0.5708-2.7799)$ & 0.6829 & - \\
\hline Malignancy & 0.1926 & $1.5597(0.8165-2.9680)$ & 0.5244 & - \\
\hline Embolus localization & 0.0248 & $2.0918(1.0812-4.0468)$ & 0.0161 & $2.3844(1.1752-4.8380)$ \\
\hline PESI class & 0.0284 & $2.0918(1.0812-4.0468)$ & 0.0048 & $2.7514(1.3610-5.5625)$ \\
\hline
\end{tabular}

FET is the Fisher exact test; logistic regression; and significant $p$ values are marked in bold.

TABLE 3: Association of in-hospital mortality and patient characteristics.

\begin{tabular}{|c|c|c|c|c|}
\hline & \multicolumn{2}{|c|}{ FET (univariate analysis) } & \multicolumn{2}{|c|}{ Logistic regression (multivariate analysis) } \\
\hline & $p$ & Odds ratio $(95 \% \mathrm{CI})$ & $p$ & Odds ratio $(95 \% \mathrm{CI})$ \\
\hline \multicolumn{5}{|l|}{ Patients without DM (154) } \\
\hline Sex & 0.2204 & $0.5402(0.2098-1.3908)$ & - & - \\
\hline Age & 0.0637 & $3.9677(0.8797-17.8963)$ & - & - \\
\hline Diabetes mellitus & - & & - & - \\
\hline Thromboembolic history & 0.7412 & $0.5657(0.1224-2.6142)$ & - & - \\
\hline Malignancy & 0.0267 & & 0.0230 & $3.1751(1.1729-8.5955)$ \\
\hline Embolus localization & 0.7946 & $0.8900(0.3182-2.4897)$ & - & - \\
\hline $\mathrm{SH}$ & 0.0061 & $4.1806(1.5082-11.5876)$ & 0.0084 & $4.0360(1.4303-11.3889)$ \\
\hline PESI class & 0.0729 & $3.9677(0.8797-17.8963)$ & & - \\
\hline \multicolumn{5}{|l|}{ All patients (190) } \\
\hline Sex & 0.5056 & $0.7084(0.3023-1.6598)$ & - & - \\
\hline Age & 0.0028 & $4.5805(1.0386-20.2016)$ & 0.1342 & $1.0397(0.9880-1.0941)$ \\
\hline Diabetes mellitus & 1.0000 & $1.0806(0.3763-3.1035)$ & - & - \\
\hline Thromboembolic history & 0.7722 & $0.7290(0.2033-2.6140)$ & - & - \\
\hline Malignancy & 0.0951 & $2.4554(1.0325-5.8388)$ & - & - \\
\hline Embolus localization & 0.8118 & $1.2613(0.4743-3.344)$ & - & - \\
\hline $\mathrm{SH}$ & 0.0092 & $3.4044(1.3488-8.5928)$ & 0.0495 & $2.5974(1.0020-6.7329)$ \\
\hline PESI class & 0.0002 & $25.6154(1.5308-428.6366)$ & 0.9968 & Indeterminate \\
\hline
\end{tabular}

FET is the Fisher exact test; logistic regression; and significant $p$ values are marked in bold.

used as a less-complicated prognostic factor for in-hospital outcomes.

Similar data were described in a recent study comparing the PESI score with a composite biomarker prognostic model that included admission glycaemia [17] and in a study analyzing the separate impacts of diabetes and SH on APErelated mortality [18].

There are some limits to this study that need to be clarified. First, as in the original PESI study, mostly categorical data (i.e., binary data for presence or absence of hypotension or tachycardia) instead of qualitative data (i.e., exact numbers for blood pressure and heart rate) were used [5]. Second, patients who have had emboli in several different branches of pulmonary artery were referred to the localization group according to the biggest branch blocked.
In this way, the total size of the PE could have been overseen, especially in patients with a large number of smaller vessels blocked. Third, patients with DM were not statistically evaluated because of the small sample size and specific issues regarding their glucose metabolism (e.g., chronic hyperglycaemia and concomitant antidiabetic medication affecting $\mathrm{SH}$ ).

\section{Conclusion}

$\mathrm{SH}$ is common in patients with APE like in a variety of other acute medical and surgical conditions. In general, $\mathrm{SH}$ is independently associated with embolus localization on MSCTA, PESI score, and in-hospital mortality. 
Considering PG is a simple test that can be measured quickly and provides additional information about clinical outcomes; it should not be overlooked in the diagnosis and prognosis estimation of many acute diseases, including APE.

\section{Data Availability}

The data used to support the findings of this study are available from the corresponding author upon request.

\section{Conflicts of Interest}

The authors declare that they have no conflicts of interest.

\section{Authors' Contributions}

All authors contributed to the study conception and design. Material preparation was performed by Velimir Altabas, Lucija Pukec, and Stella Mlinarić. Data collection was performed by Hrvoje Pintarić and Aljoša Šikić. Analysis was performed by Velimir Altabas, Lucija Pukec, and Stella Mlinarić. The first draft of the manuscript was written by Velimir Altabas, Lucija Pukec, and Stella Mlinarić, and all authors commented on previous versions of the manuscript. All authors read and approved the final manuscript.

\section{Acknowledgments}

The authors would like to thank Ms. Kathleen Banjac for English editing of the text. This research did not receive any specific grant from any funding agency in the public, commercial, or not-for-profit sector. However, article processing charges will be covered by Boehringer Ingelheim Zagreb d. o. o.

\section{References}

[1] A. K. Tarbox and M. Swaroop, "Pulmonary embolism," International Journal of Critical Illness and Injury Science, vol. 3, no. 1, pp. 69-72, 2013.

[2] J. A. Heit, "Epidemiology of venous thromboembolism," Nature Reviews Cardiology, vol. 12, no. 8, pp. 467-474, 2015.

[3] M. A. M. Rogers, D. A. Levine, N. Blumberg, S. A. Flanders, V. Chopra, and K. M. Langa, "Triggers of hospitalization for venous thromboembolism," Circulation, vol. 125, no. 17, pp. 2092-2099, 2012.

[4] H. Dogan, A. de Roos, J. Geleijins, L. J. M. Huisman, and L. Kroft, "The role of computed tomography in the diagnosis of acute and chronic pulmonary embolism," Diagnostic and Interventional Radiology, vol. 21, no. 4, pp. 307-316, 2015.

[5] B. Long and A. Koyfman, "Best clinical practice: controversies in outpatient management of acute pulmonary embolism," The Journal of Emergency Medicine, vol. 52, no. 5, pp. 668-679, 2017.

[6] P. E. Marik and R. Bellomo, "Stress hyperglycemia: an essential survival response!” Critical Care, vol. 17, no. 2, p. 305, 2013.

[7] N. Scherz, J. Labarère, D. Aujesky, and M. Méan, "Elevated admission glucose and mortality in patients with acute pulmonary embolism," Diabetes Care, vol. 35, no. 1, pp. 25-31, 2012.
[8] F. Farrokhi, D. Smiley, and G. E. Umpierrez, "Glycemic control in non-diabetic critically ill patients," Best Practice \& Research Clinical Endocrinology \& Metabolism, vol. 25, no. 5, pp. 813-824, 2011.

[9] R. A. Defronzo, "From the triumvirate to the ominous octet: a new paradigm for the treatment of type 2 diabetes mellitus," Diabetes, vol. 58, no. 4, pp. 773-795, 2009.

[10] C. S. Rau, S. C. Wu, Y. C. Chen et al., "Higher mortality in trauma patients is associated with stress-induced hyperglycemia, but not diabetic hyperglycemia: a cross-sectional analysis based on a propensity-score matching approach," International Journal of Environmental Research and Public Health, vol. 14, no. 10, p. 1161, 2017.

[11] L. Leonidou, M. Michalaki, A. Leonardou et al., "Stress-induced hyperglycemia in patients with severe sepsis: a compromising factor for survival," The American Journal of the Medical Sciences, vol. 336, no. 6, pp. 467-471, 2008.

[12] V. Altabas, K. Altabas, M. Berković-Cigrovski, S. Malosevac, M. Vrkljan, and V. Nikolić Heitzler, "Glucose metabolism disorders in patients with acute coronary syndromes," Acta Clinica Croatica, vol. 51, no. 1, pp. 71-77, 2012.

[13] E. Olariu, N. Pooley, A. Danel, M. Miret, and J. C. Preiser, "A systematic scoping review on the consequences of stress-related hyperglycaemia," PLoS One, vol. 13, no. 4, Article ID e0194952, 2018.

[14] The Committee of the Japan Diabetes Society, Y. Seino, and K. Nanjo, "Report of the committee on the classification and diagnostic criteria of diabetes mellitus," Journal of Diabetes Investigation, vol. 1, no. 5, pp. 212-228, 2010.

[15] M. Kosiborod, S. S. Rathore, S. E. Inzucchi et al., “Admission glucose and mortality in elderly patients hospitalized with acute myocardial infarction," Circulation, vol. 111, no. 23, pp. 3078-3086, 2005.

[16] D. Aujesky, D. S. Obrosky, R. A. Stone et al., "Derivation and validation of a prognostic Model for pulmonary embolism," American Journal of Respiratory and Critical Care Medicine, vol. 172, no. 8, pp. 1041-1046, 2005.

[17] J. Lee, J. Lee, J. Huh et al., "Prognostic value of blood biomarkers in patients with unprovoked acute pulmonary embolism," Annals of Thoracic Medicine, vol. 14, no. 4, pp. 248-253, 2019.

[18] A. Akirov, A. Grossman, T. Shochat, and I. Shimon, "Blood glucose on admission and mortality in patients with venous thromboembolism," Journal of Diabetes and Its Complications, vol. 31, no. 2, pp. 358-363, 2017.

[19] M. Shimon, H. Uyarel, S. Avsar et al., "Admission glucose level predicts in-hospital mortality in patients with acute pulmonary embolism who were treated with thrombolytic therapy," Lung, vol. 194, no. 2, pp. 219-226, 2016.

[20] C. Risk, C. W. Chia, J. M. Egan, and L. Ferrucci, "Age-related changes in glucose metabolism, hyperglycemia, and cardiovascular risk," Circulation Research, vol. 123, no. 7, pp. 886-904, 2018. 